\title{
la construcción mixta ante los nuevos métodos de análisis y criterios de seguridad
}

JULIO MARTINEZ CALZON y JESUS ORTIZ HERRERA, Dres. Ingenieros de Caminos $569-17$

\section{sinopsis}

Se ofrece en este artículo una vîsión sîntética, îlustrada mediante ejemplos seleccionados, de la interacción entre los nuevos criterios de seguridad y la concepción tipológica y constructiva de las secciones y estructuras mixtas; $y$, por otro lado, entre los nuevos métodos de análisis y los medios de cálculo electrónico actualmente disponibles.

\section{INTRODUCCION}

El objeto de este artículo es

el de comentar la evolución y posibilidades de la construcción mixta ante los actuales métodos de análisis y criterios de seguridad, bajo distintas facetas:

- Se incluye una breve visión panorámica del planteamiento y alcance de estos nuevos métodos y criterios.

- Se resalta y ratifica mediante ejemplos la interacción entre los mismos y la concepción tipológica y constructiva de las estructuras mixtas, mostrando cómo, por un lado, los nuevos criterios de seguridad han posibilitado la transformación y creación de tipologías y procesos constructivos; y, por otro, cómo la complejidad de estos nuevos sistemas ha exigido (y continuará, sin duda, exigiéndolo) el desarrollo de nuevas teorías y métodos potentes para su análisis.
- Se comenta asimismo la dialéctica existente entre los nuevos planteamientos y formalismos teóricos con los medios de cálculo automático, ofreciendo una visión concisa, e ilustrada con ejemplos seleccionados, del alcance actual de la aplicación de los métodos "computerizados" a la construcción mixta.

Los diferentes conceptos y algoritmos aludidos a lo largo de estas líneas, pueden encontrarse extensamente desarrollados en la obra:
"Construcción mixta hormigón-acero» (1), a la que se ha intentado dotar del máximo alcance y proyección actual y futura, pero en la que las ideas fundamentales antes señaladas, o no han encontrado una cabida adecuada en la citada obra (por corresponder más bien a un "informe", o visión actualizada de un tema sin preocupación por la vigencia prolongada en el tiempo de las afirmaciones en él vertidas), o se encuentran dispersas en el necesario detalle de una obra exhaustiva; si bien de la misma pueden igualmente extraerse las conclusiones fundamentales aquí expuestas, ello sería indudablemente tras un proceso más largo y que se trata de facilitar con estas líneas.

(1) Ed. Rueda, 1978 (por los mismos autores que suscriben este artículo). 


\section{ALCANCE ACTUAL Y ENFOQUE DE LAS COMPROBACIONES TENSIONALES}

Debido a la generalización de los métodos de cálculo en agotamiento, cuyo interés y alcance se comentan en el siguiente apartado, las comprobaciones tensionales han pasado en cierto modo a una categoría de comprobaciones secundarias, justificativas del adecuado comportamiento $y$ funcionalidad en servicio de la pieza o estructura, cuya capacidad resistente se controla y garantiza mediante otros procedimientos. Sólo excepcionalmente los métodos elásticos o lineales deben ser utilizados para controlar estados límites últimos:

- Para secciones mixtas cuya sección parcial de acero estructural presente esbelteces elevadas no es posible la aplicación directa de los criterios elastoplásticos usuales, requiriendo éstos adaptaciones profundas (definición de secciones "eficaces" de cálculo, o utilización de diagramas tensión-deformación "aparentes" de las chapas metálicas comprimidas); alternativamente, en defecto del control del estado límite de agotamiento conforme a dichos criterios, cabe calcular este tipo de secciones elásticamente, por métodos de tensiones admisibles y observando criterios "clásicos" de seguridad frente a la abolladura de las chapas.
- En el caso de piezas sometidas a la acción de sobrecargas móviles importantes y de alta repetitividad, es preciso controlar, además de los estados límites usuales (de agotamiento y servicio), el estado límite de fatiga en los distintos materiales de la sección y en los conectadores de la pieza. Puesto que el fenómeno de la fatiga es susceptible de producir la rotura, es claro que se trata de un estado límite último; sin embargo, su control se efectúa a partir de distribuciones tensionales obtenidas por métodos lineales bajo las solicitaciones características 0 típicas, y limitando dichas tensiones en función de la resistencia característica a la fatiga bajo un número de ciclos de carga adecuadamente mayorado (o bajo el número característico de repeticiones de carga estimado durante la vida esperada de la estructura, y minorando adecuadamente la correspondiente resistencia a la fatiga).

Salvo en estos casos, la capacidad resistente de las piezas mixtas está suficientemente garantizada a través del control del estado límite de agotamiento por métodos elastoplásticos o rígido-plásticos, y la misión de los controles tensionales de servicio se reduce a los siguientes objetivos:

- Asegurar la validez de los métodos lineales o cuasilineales normalmente utilizados para el control de los estados límites de servicio (flechas, fisuración, vibraciones, etc.), que de otro modo deberían estudiarse por métodos no lineales.
- Asegurar que la pérdida de linealidad se produce sólo bajo las cargas mayoradas y no bajo las cargas características, de tal forma que el estudio en régimen no lineal bajo las acciones mayoradas pueda efectuarse suponiendo un comportamiento noval o prácticamente noval de los materiales; y de que en servicio no se produzcan plastificaciones intermitentes, que podrían dar lugar a una acumulación excesiva de deformaciones plásticas remanentes o incluso producir una rotura prematura por fatiga en régimen plástico. El control de que estos fenómenos no se producen sólo puede obviarse en el caso de piezas con cargas estáticas o predominantemente estáticas.

Por estas razones se mantiene la necesidad de limitar en la situación de servicio las tensiones de los materiales por debajo de sus límites elásticos o resistentes ("estado límite de plastificación"), con un pequeño margen que garantice el práctico mantenimiento del carácter lineal en servicio, muy por encima de las tensiones admisibles "clásicas". 
En cuanto al enfoque práctico de los cálculos tensionales de servicio, cabe recomendar como método suficientemente preciso (en relación a su alcance estrictamente de control de servicio) y eminentemente intuitivo y cómodo, el método de la sección ideal de acero, adecuadamente adaptado para la consideración de los fenómenos "cuasilineales" de las secciones mixtas (deformaciones diferidas y fisuración del hormigón):

- Para la consideración de la fluencia del hormigón, es suficiente actuar sobre el coeficiente de equivalencia de dicho material, en forma sobradamente conocida; pero se recomienda, en orden a mejorar la calidad de los cálculos, adoptar coeficientes de fluencia "aparentes", de expresión directa, que permiten considerar de modo aproximado los fenómenos de fluencia variable que ocurren en las secciones mixtas.

- Para los cálculos tensionales en régimen de fisuración, es preciso determinar la sección "virtual" de cálculo, bien iterando sobre la posición de la fibra neutra de tensiones, o bien mediante fórmulas fácilmente deducibles para secciones standard. Sin embargo, cuando la fisuración interactúa con los fenómenos de fluencia y con presolicitaciones aplicadas a diferentes secciones parciales (piezas mixtas complejas), sólo algoritmos apropiados para su programación en ordenador pueden dar una respuesta correcta a este tipo de problemas, aunque caben simplificaciones para su resolución aproximada en muchos casos prácticos.

\section{PLANTEAMIENTO DEL CALCULO EN AGOTAMIENTO}

Los métodos de cálculo elástico o de tensiones admisibles han constituido durante mucho tiempo el único procedimiento de control de las estructuras mixtas, como lo fueron en su tiempo para otros esquemas estructurales; y se completaban con criterios apropiados para introducir las variaciones diferidas producidas por las características reológicas del hormigón: la retracción y la fluencia bajo solicitaciones permanentes.

Sin embargo, puede decirse que, en general, este método es totalmente inapropiado para los sistemas mixtos, dado que en los mismos se combinan materiales con diagramas tensión-deformación y comportamientos diferidos pronunciadamente diferentes $y$, por consiguiente, solamente con un extraordinario cuidado en el diseño y disposición relativa de dichos materiales podía llegarse a obtener rendimientos cercanos a los óptimos (situaciones que se denominarían estrictamente críticas).

Por el contrario, otros métodos de cálculo, fundamentados básicamente en el comportamiento global real de los materiales, mediante procedimientos no lineales, permiten aprovechar con total garantía - tanto funcional o de servicio, como de agotamiento o seguridad- las profundas redistribuciones de trabajo resistente que se producen entre los diversos materiales que conforman las piezas y secciones mixtas.

Como ejemplo meramente ilustrativo, aunque no inusual y rebuscado, sino perteneciente a la construcción mixta ordinaria, puede presentarse el caso siguiente:

Capacidad de utilización de una pieza mixta isostática de $16 \mathrm{~m}$ de luz, sometida a cargas permanentes y sobrecargas uniformemente repartidas (fig. iit.1).
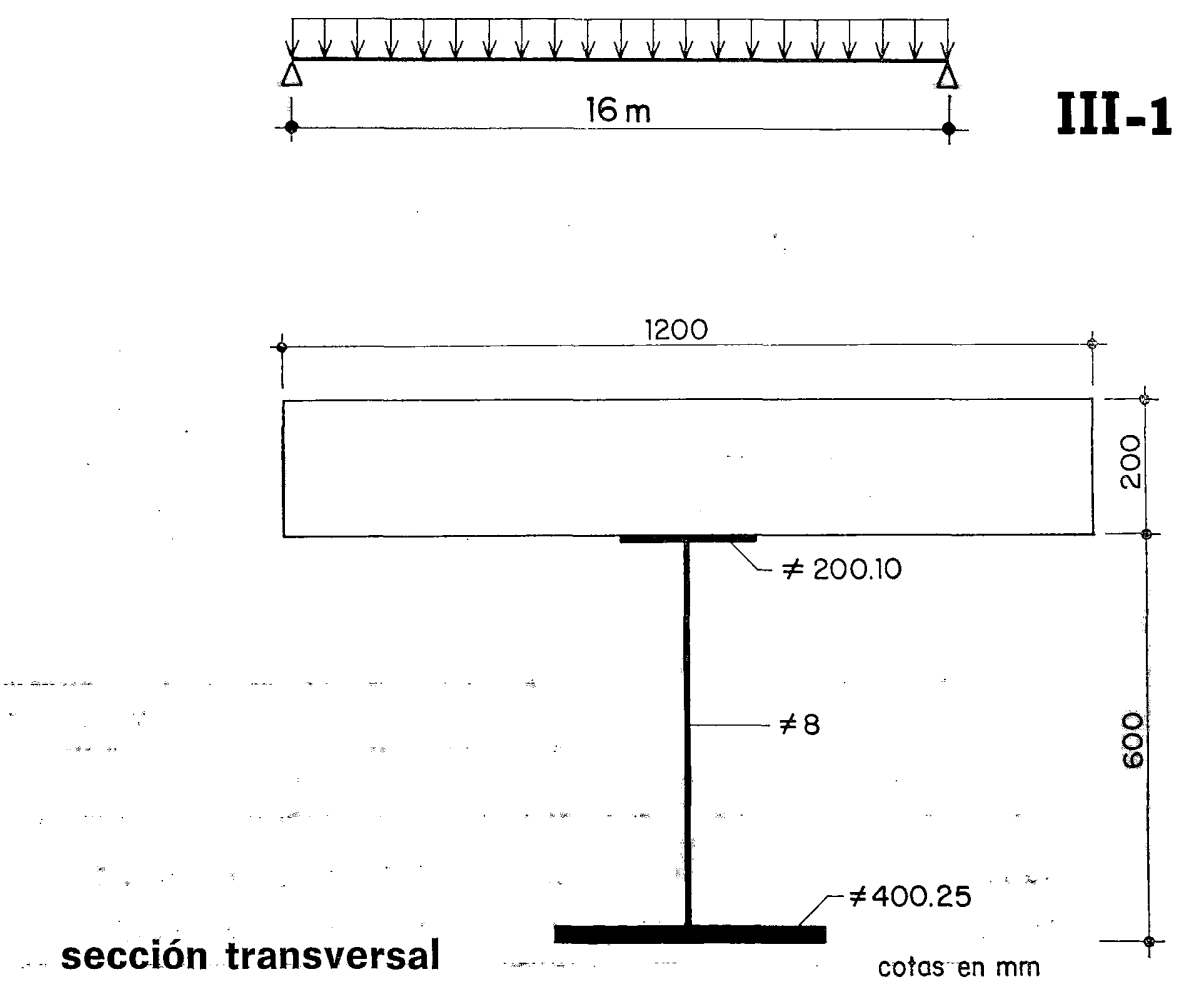
Cargas permanentes:

$$
\begin{aligned}
& \text { Peso propio } \simeq 0,78 \mathrm{t} / \mathrm{m} . \mathrm{I} . \quad ; \quad M_{\mathrm{PP}}=25 \mathrm{mt} \text { en centro luz. } \\
& \text { Carga muerta } \simeq 0,78 \mathrm{t} / \mathrm{m} . \mathrm{l} . \quad ; \quad M_{\mathrm{CM}}=25 \mathrm{mt} \text { en centro luz. }
\end{aligned}
$$

Materiales:

$$
\begin{aligned}
\text { Acero: } A 42 b ; & f_{\mathrm{yk}}=2.600 \mathrm{kp} / \mathrm{cm}^{2} . \\
\text { Hormigón: } f_{\mathrm{ck}}= & 250 \mathrm{~kg} / \mathrm{cm}^{2} . \text { Retracción final: } \varepsilon^{\prime} \mathrm{cs}_{\infty}=20 \times 10^{-5} . \\
& \text { Coeficiente final de fluencia } \varphi_{\infty}=2 .
\end{aligned}
$$

Análisis elástico:

$\sigma_{\text {adm }}$ hormigón $=100 \mathrm{kp} / \mathrm{cm}^{2}$

$\sigma_{\text {adm }}$ acero $=1.735 \mathrm{kp} / \mathrm{cm}^{2}$
Análisis en rotura:

$$
\begin{aligned}
& \gamma_{\mathrm{c}}=1,5 \\
& \gamma_{\mathrm{s}}=1,0 \\
& \gamma_{\mathrm{f}}=1,5
\end{aligned}
$$

En la figura III.2 se esquematizan los estados tensionales correspondientes a las situaciones de esta pieza, en dos procesos constructivos diferentes:

a) Pieza metálica autoportante para el peso propio.

b) Pieza metálica apeada durante la ejecución,

$y$ en los instantes inicial $t=t_{0}$, y final $t \rightarrow \infty$, cuando se pueden considerar terminadas la retracción y la fluencia.
Se indican en la misma figura los valores de los momentos flectores en centro de luz debidos a la sobrecarga $\left(\mathrm{M}_{\mathrm{sc}}\right)$ que provocan en una fibra el valor de la tensión admisible, y los correspondientes momentos totales $(M)$ de cargas permanentes y sobrecarga.
Para el caso en que la sección se calcula en rotura, se indican, asimismo, las distribuciones tensionales que se producen en servicio, bajo el correspondiente momento de utilización ( $\left.M=M_{\mathrm{pl}, \mathrm{d}} / \gamma_{\mathrm{f}}\right)$, señalándose, asimismo, el correspondiente momento debido a la sobrecarga $\left(\mathrm{M}_{\mathrm{sc}}\right)$, así como las relaciones ( $\alpha$ y $\alpha_{\mathrm{SC}}$ ) de ambos valores a los ante-

\begin{tabular}{|c|c|c|c|c|c|}
\hline \multirow{2}{*}{ CASO } & \multirow{2}{*}{ METODO ROTURA } & \multicolumn{4}{|c|}{ METODO ELASTICO } \\
\hline & & \multicolumn{2}{|c|}{$t=t_{0}$} & \multicolumn{2}{|c|}{$\mathbf{t} \rightarrow \infty$} \\
\hline No apeado & $3,02 \mathrm{t} / \mathrm{m} . \mathrm{l}$ & 2,25 t/m.l.; & $\alpha_{S C}=1,34$ & $0,69 \mathrm{t} / \mathrm{m} . \mathrm{I} . ;$ & $\alpha_{S C}=4,39$ \\
\hline Apeado & $3,02 \mathrm{t} / \mathrm{m} . \mathrm{I}$. & 1,80 t/m.l.; & $\alpha_{\mathrm{SC}}=1,68$ & 2,10 t/m.l.; & $\alpha_{\mathrm{SC}}=1,44$ \\
\hline
\end{tabular}
riormente indicados como válidos según el método de las tensiones admisibles.

En el cuadro siguiente se indican también los valores y las sobrecargas uniformes de uso para cada caso: 
DISTRIBUCION DE TENSIONES EN LA SECCION MIXTA PARA LOS DIVERSOS CASOS, ESTADOS Y CRITERIOS CONSIDERADOS

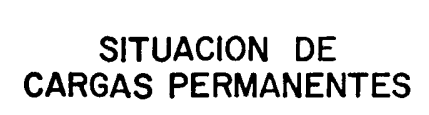

SITUACION DE SERVICIO

(cargas permanentes + sobrecargas de uso)

\section{III-2}

METODO ELASTICO METODO DE ESTADOS LIMITES
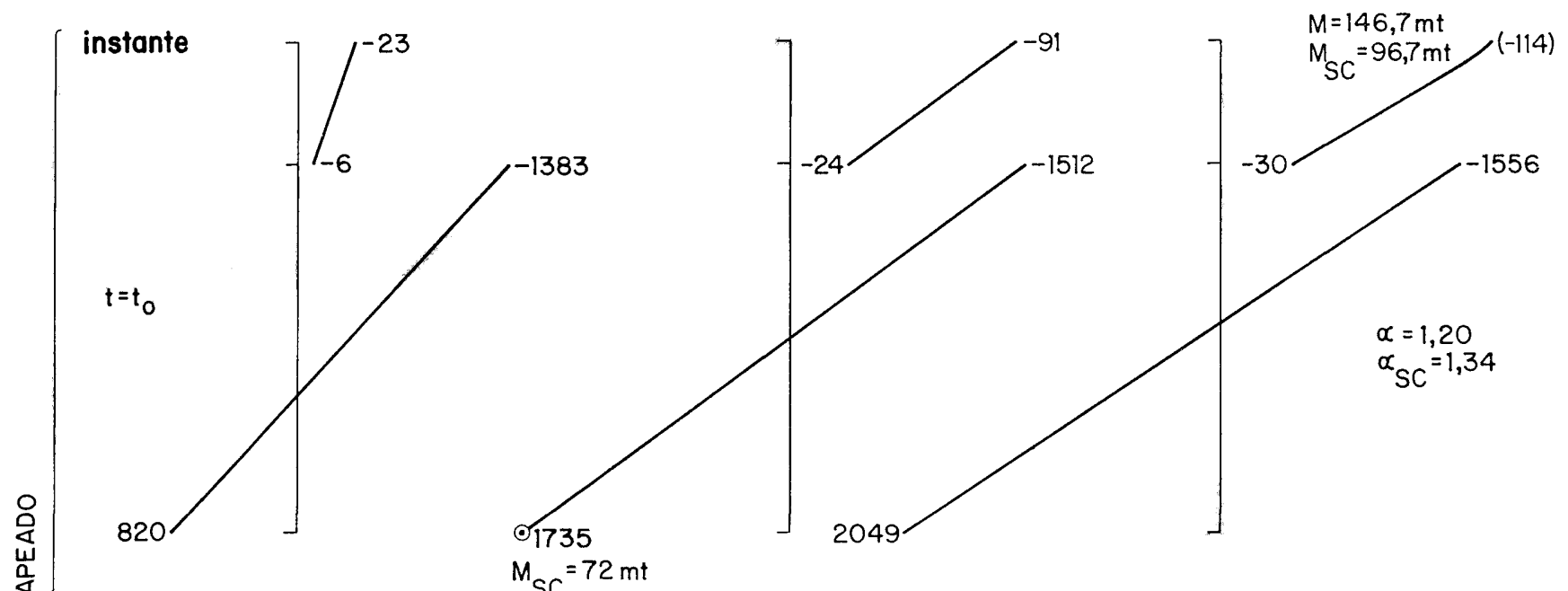

은

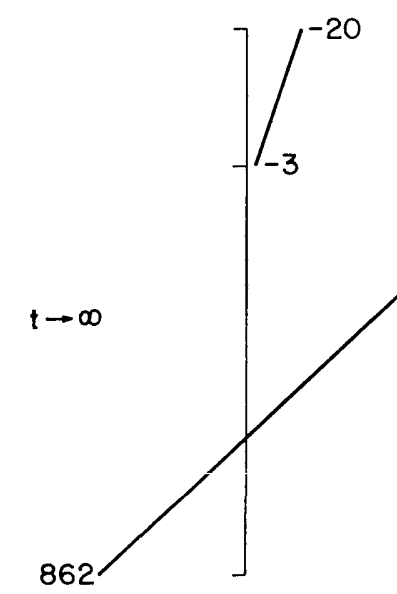

$M=122 \mathrm{mt}$
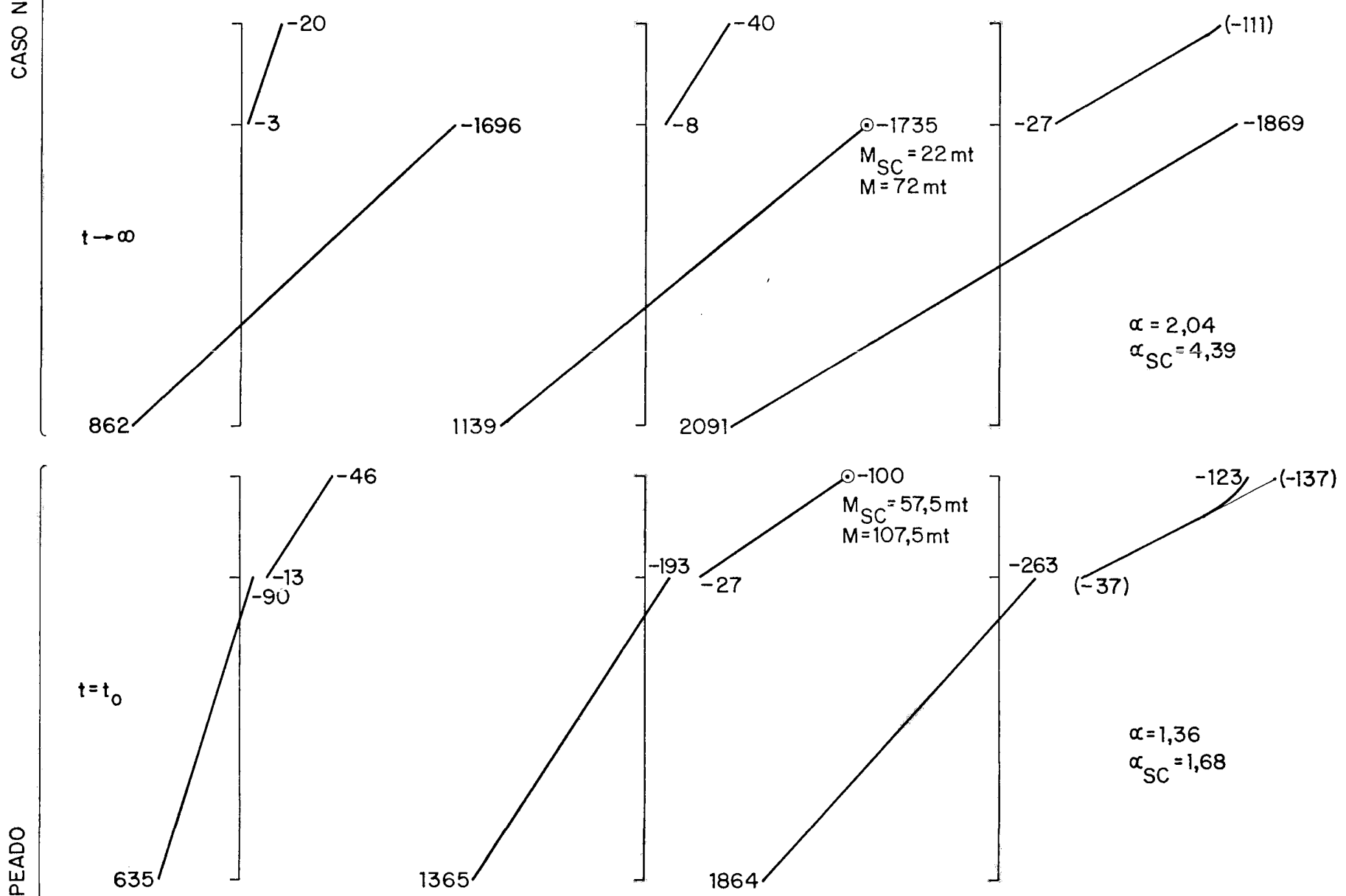

通
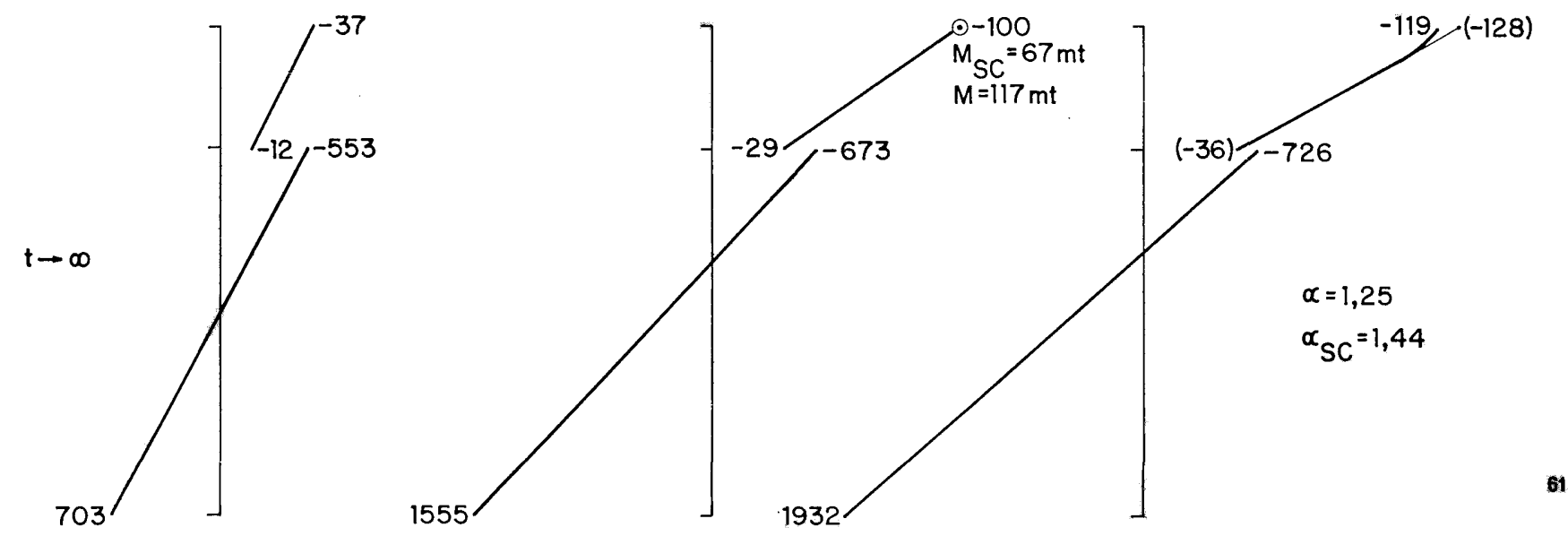


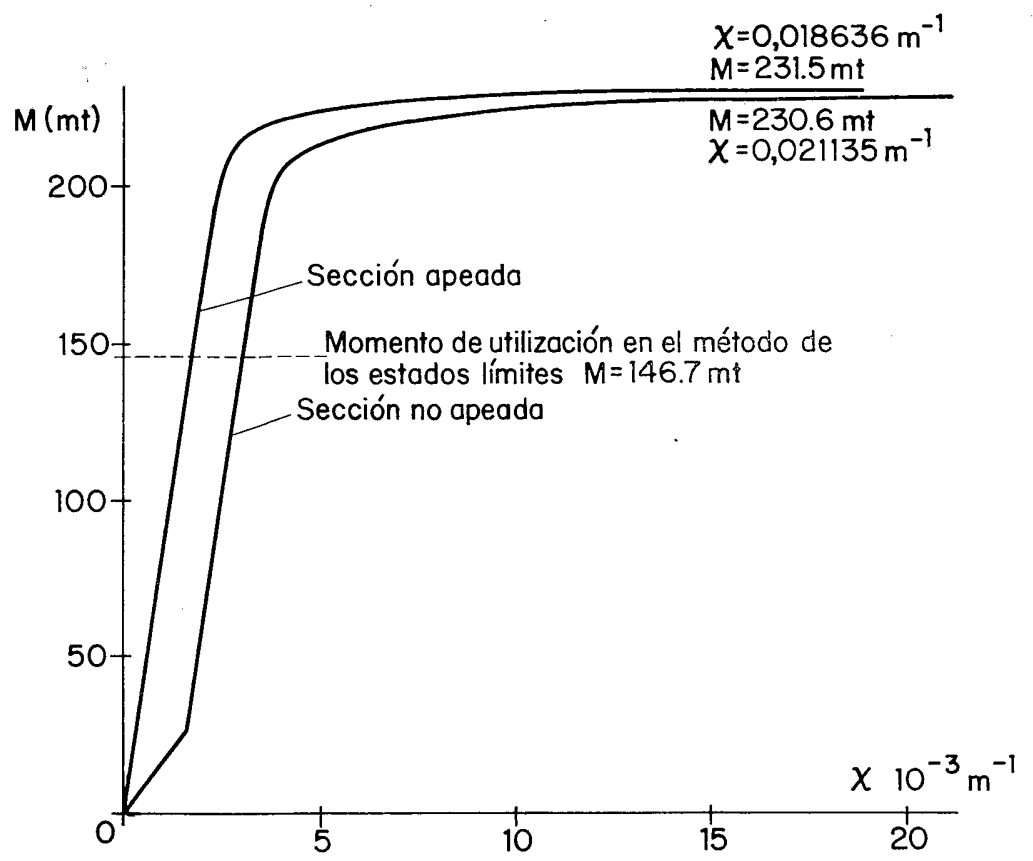

III-3

Como puede verse, las diferencias de aprovechamiento son notables, sobre todo en el caso no apeado, y si se observa (fig. III.3) que la situación de servicio obtenida mediante el cálculo en rotura se encuentra, para ambos casos constructivos, perfectamente dentro de la rama prácticamente elástica del diagrama momentos-curvaturas, y que los valores del coeficiente de seguridad global de uno y otro caso son 1,57 y 1,58 , puede concluirse la perfecta validez del procedimiento.

Así pues, los métodos no lineales se presentan como enormemente favorables para la mejor utilización y desarrollo de los sistemas mixtos, pero no sólo a nivel de secciones, sino de forma también muy favorable a nivel de estructuras, dado que la construcción mixta tiene como característica esencial $\mathrm{poder}$ graduar ampliamente el grado de empotramiento relativo entre piezas, mediante la apropiada distribución de los diferentes materiales, sin las secuelas que este tratamiento produce en los sistemas de hormigón armado o pretensado, en los cuales las redistribuciones de momentos hiperestáticos se limitan apreciablemente para evitar roturas prematuras por flexión (agotamiento de la limitada capacidad de deformación plástica) y/o desfavorables condiciones de comportamiento a cortante (fisuración diagonal excesiva); y en los entramados m e táli c o s traslacionales, comprobaciones rigurosas de la estabilidad de conjunto de los sistemas plastificados, dado que la presencia de rótulas reduce apreciablemente la rigidez global del sistema, mientras que en la construcción mixta es posible mejorar este comportamiento seleccionando adecuadamente la tipología de los elementos verticales.

Pero el análisis no lineal de estructuras mediante métodos rigurosos basados en la definición sección a sección de los correspondientes diagramas momentos-curvaturas, no puede emplearse salvo en casos muy sencillos, y es preciso entonces adoptar criterios simplificadores, tanto en el estudio de las estructuras (idealización de rótulas plásticas, no consideración de las deformaciones entre rótulas, etcétera), como en el de
Diagramas momentos-curvaturas considerando para los materiales resistencias características.

las secciones (cálculo rígidoplástico de momentos de agotamiento; criterios aproximados para la estimación de reducciones por interacción entre esfuerzos, etc.).

En relación a los métodos elásticos, los métodos no lineales presentan marcados inconvenientes prácticos, tales como la invalidez del principio de superposición, dificultad apriorística de definición rigurosa de secciones, etc.; lo cual, en la actualidad, en tanto no se incrementen aún más la velocidad y capacidad del cálculo de los métodos electrónicos, hacen prácticamente inviables estos procedimientos de modo exclusivo o sistemático. No obstante, pueden paliarse estas consideraciones mediante tratamientos rigurosos de zonas parciales y configuraciones dadas de cargas, que lleven a obtener coeficientes de redistribución apropiados para el resto de la estructura $y$ de las combinaciones de acciones.

Todo lo anteriormente expuesto lleva a considerar como procedimiento de cálculo más apropiado de las estructuras mixtas el denominado método de los estados límites, que manteniendo los criterios de cálculo elástico de la estructura, complementados con adecuados coeficientes de redistribución por fisuración y plasticidad (establecidos genéricamente por métodos no lineales rigurosos), permiten emplear con toda utilidad los métodos no lineales o en rotura de las secciones mixtas, incluyendo en los mismos las condiciones de interacción de esfuerzos simultáneos, reducciones por inestabilidad local (abolladura) y otras posibles, establecidas con toda rigurosidad. 


\section{DIALECTICA ENTRE TEORIA Y PRACTICA}

Los métodos de cálculo en agotamiento, esbozados en el apartado anterior, no solamente han llevado a utilizar con mayor rendimiento las soluciones que podríamos denominar tradicionales de la construcción mixta, sino que el conocimiento de nuevos métodos ha dado lugar a la introducción de nuevas combinaciones de materiales en las secciones; y a partir de éstas, nuevas tipologías de las estructuras y nuevos criterios y sistemas constructivos.
Todo ello puede apreciarse con sencillez en el caso del ejemplo anterior, en el cual si se utilizan métodos de tensiones admisibles, la sección debería ser apeada durante el proceso constructivo, dado que de otra forma la sobrecarga de utilización sería muy reducida; mientras que si se analiza por el método de estados límites las condiciones constructivas no influyen prácticamente y basta comprobar que en la fase de servicio no se producen condiciones desfavorables $\left(\sigma_{\mathrm{s}} \geq 0,95 \mathrm{f}_{\mathrm{yk}}\right.$; abolladuras; etcétera) y que durante la fase de montaje autoportante se mantenga una seguridad suficiente frente a la inestabilidad por pandeo lateral. Cumpliéndose estos requisitos puede perfectamente prescindirse de dicho apeo, lo cual en muchos casos constructivos resulta decisivo.
A efectos funcionales, las piezas metálicas deben construirse con las oportunas contraflechas de ejecución, en orden a conseguir una forma adecuada de las superficies de uso y una correcta apariencia estética si la propia pieza es visible. Como orden de magnitud apropiado puede estimarse que para el total de cargas permanentes más un tercio de la sobrecarga de uso la pieza se encuentre prácticamente horizontal.

En el cuadro siguiente se exponen las flechas de los diferentes estados analizados en el ejemplo, y las contraflechas requeridas en el caso de análisis en estados límites, si se quiere cumplir la condición antedicha:

\section{FLECHAS (en cm)}

\begin{tabular}{|c|c|c|c|c|c|}
\hline \multirow[b]{2}{*}{ CASO } & \multirow[b]{2}{*}{ INSTANTE } & \multicolumn{2}{|c|}{ METODO ELASTICO } & \multicolumn{2}{|c|}{ METODOS EN ESTADOS LIMITES } \\
\hline & & Carga permanente & $\begin{array}{l}\text { Carga permanente } \\
\text { más sobrecarga } \\
\text { límite }\end{array}$ & $\begin{array}{l}\text { Carga permanente } \\
\text { más sobrecarga } \\
\text { de uso }\end{array}$ & $\begin{array}{c}\text { Contraflecha } \\
\text { para } \mathbf{C P}+\mathbf{S C} / 3\end{array}$ \\
\hline \multirow{2}{*}{ No apeado } & $\mathbf{t}=\mathbf{t}_{0}$ & 4,66 & 6,87 & 7,63 & \multirow{2}{*}{6,47} \\
\hline & $\mathbf{t} \rightarrow \infty$ & 5,49 & 6,17 & 8,46 & \\
\hline \multirow{2}{*}{ Apeado } & $\mathbf{t}=\mathbf{t}_{0}$ & 1,53 & 3,30 & 4,50 & \multirow{2}{*}{3,72} \\
\hline & $t \rightarrow \infty$ & 2,73 & 4,78 & 5,70 & \\
\hline
\end{tabular}


Es decir, el cambio en el método de cálculo permite prescindir de la disposición de apeos, dado que el mismo garantiza que tanto la seguridad como la funcionalidad son correctas; de lo único que se prescinde es de aplicar el concepto ya superado de tensión admisible como control de la seguridad, válido en ausencia de otros controles, pero prácticamente inoperante para la utilización racional de los sistemas mixtos (1).

Otros ejemplos que permiten ratificar lo indicado al principio de este apartado son la práctica inutilidad de todos los procedimientos de presolicitación por incurvación, preflexión o predeformación que no incorporen material resistente interno en las secciones, dado que su único fin consistía en establecer estados tensionales previos de tal forma que los niveles admisibles requeridos se mantuvieran en forma adecuada. No teniendo necesidad de controlar esta circunstancia, $y$ al no aumentar la capacidad resistente última (2), su empleo resulta prácticamente inapropiado. Solamente en casos muy especiales en los que el comportamiento en fase de servicio pudiera ser desfavorable pueden llegar a ser adecuados, pero incluso

(1) Subsiste la limitación tensional del «estado límite de plastificación" como garantía frente a fenómenos generalmente no analizables con sencillez (v. ap. II). No obstante, si se llegan a conocer, o se aplican criterios para valorar la incidencia de estos posibles fenómenos, conceptualmente la limitación señalada podría eliminarse.

(2) Solamente en ciertos casos en los que la condición básica radica en la inestabilidad por abolladura de los componentes metálicos, podrían conseguirse incrementos apreciables de la capacidad resistente. en tales casos suele resultar preferible un ligero incremento de materiales que la introducción en la construcción de procesos complicados y prácticamente sin incidencia en la resistencia última.

Abierta la puerta al empleo de materiales de muy variadas condiciones resistentes, puede preverse un interesante incremento y modificaciones en la utilización y posibilidades de los sistemas mixtos, en base a la imaginación y creatividad de nuevos procesos. A título informativo pueden exponerse diversos sistemas que, incorporando materiales diversos permiten favorables soluciones:

\section{A) Hormigón inferior en pie-} zas en cajón (fig. IV.1). Utilizando métodos de tensiones admisibles su empleo sería prácticamente inútil, dado que para mantener la tensión del hormigón en valores admisibles las tensiones del acero serían claramente muy inferiores a su respectivo límite de utilización. Por el contrario, cuando la seguridad frente al agotamiento se controla adecuadamente (ya sea por el método elastoplástíco o el rígido-plástico), en la situación de servicio se pueden considerar en el hormigón tensiones notablemente superiores a las tensiones admisibles clásicas, aunque resulta siempre conveniente controlar que no se produce una acusada pérdida de linealidad en dichas situaciones de servicio.
B) Empleo de secciones metálicas híbridas (figura IV.2). El aprovechamiento completo de las partes de acero de resistencia elevada solamente $\mathrm{pu}$ u de conseguirse con estados de tensión superiores a los denominados admisibles en las restantes zonas de acero.

C) Secciones mixtas armadas (fig. IV.3). Las tensiones admisibles en la fibra superior de acero estructural limitarían el empleo de las armaduras de alta resistencia.

D) Secciones NEX-TEN (figura IV.4). La combinación de materiales de muy diverso tipo solamente puede optimizarse con criterios no lineales.

E) Seccíones tipo 3E (figura IV.5). Es válido el mismo comentario indicado para el caso anterior.

La utilización de los nuevos métodos de cálculo posibilita, como se ha visto, la eficaz combinación de muy diversos materiales en las secciones, lo cual eleva notablemente el grado de complejidad de las secciones y piezas, como también puede observarse; todo ello conduce a la necesidad de efectuar el análisis mediante un tratamiento general en ordenador con algoritmos y programas apropiados, que no solamente permiten reducir el tiempo absoluto de cálculo, sino que proporcionan al técnico que los utiliza una rápida generación de experiencia e intuición del complejo comportamiento de tales sistemas. 


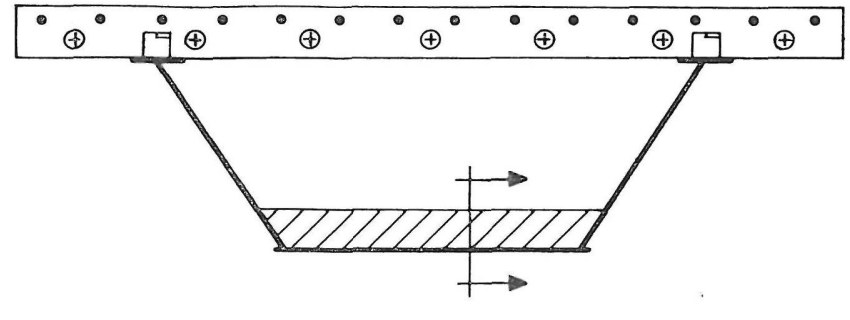

IV=1 Viga mixta en cajón con hormigón inferior.

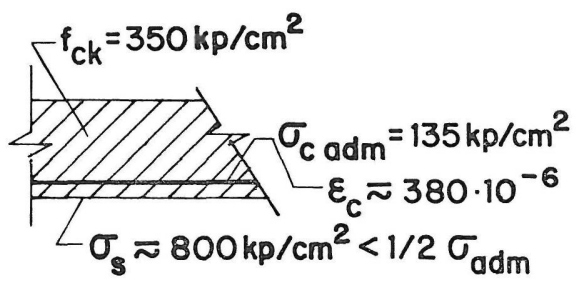

Situación en caso de tensiones admisibles.

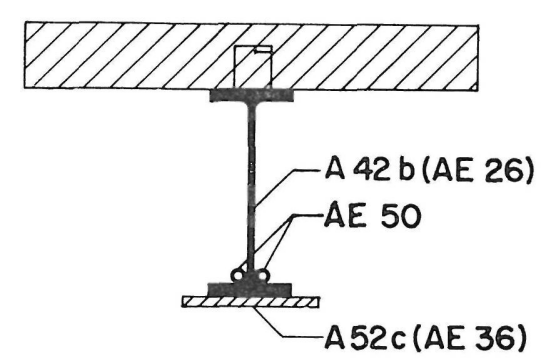

IV-2 viga mixta con sección metálica híbrida.

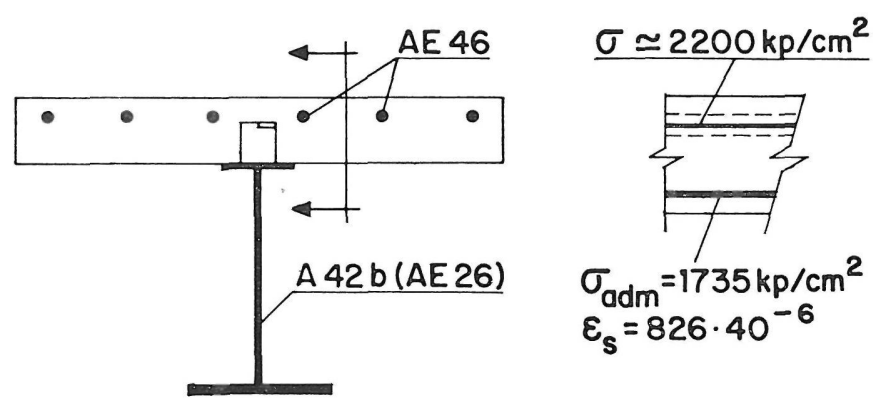
TV-3 $\begin{aligned} & \text { Viga mixta con cabeza de hormigón armado, en } \\ & \text { zonas con momentos negativos. }\end{aligned}$

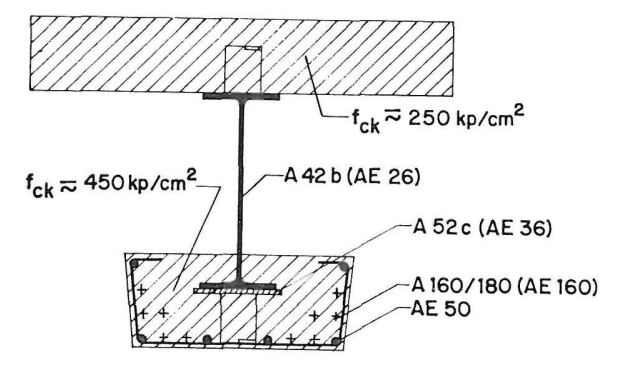

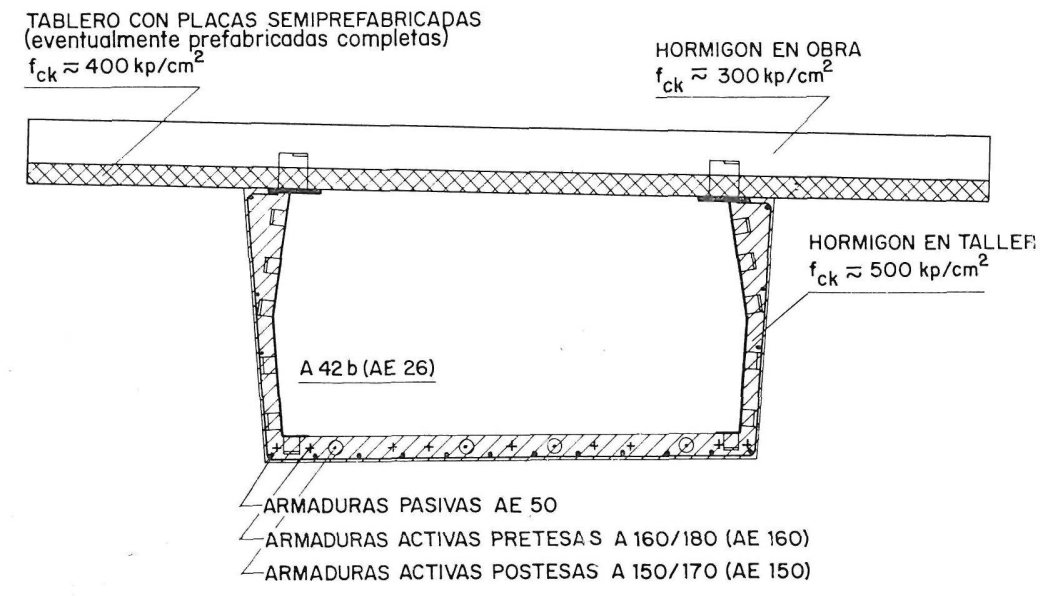

$I V=4 . \begin{aligned} & \text { Pieza NEX-TEN con pretesado (y aventualmente } \\ & \text { postesado) del hormigóln inferior. }\end{aligned}$

$I V=4 \quad \begin{aligned} & \text { Pieza NEX-TEN con pretesado }(y \text { av } \\ & \text { postesado) del hormigóln inferior. }\end{aligned}$ IV=5 lieza Sistema 3E con elementos metálicos de 
Asimismo, la proliferación de materiales, procesos constructivos, interacciones, y en general toda la temática de las secciones y piezas mixtas modernas, determinan la imposibilidad práctica de operar con fórmulas, tablas o ábacos, dado el gran número de parámetros que se manejan; este tipo de planteamientos podría resultar todavía válido para sistemas mixtos estrictamente convencionales, pero la complejidad de los nuevos sistemas desborda generalmente la capacidad de información suministrable con un volumen razonable de letra impresa. Ello lleva inevitablemente a nuevos procedimientos de estudio y cálculo basados en el empleo sistematizado de formulaciones algorítmicas apropiadas para su empleo en ordenador y máquinas programables. Esta transformación, que aquí se señala para el tratamiento $y$ estudio de las construcciones mixtas en sustitución de las tradicionales fórmulas, ábacos o tablas, se extiende realmente a todo el ámbito de las actividades técnicas del hombre como necesidad frente a la creciente complejidad del medio y las relaciones humanas.

$Y$ del adecuado manejo de estos algoritmos, combinados con las condiciones de rapidez, conversacionalidad y respuesta codificada en curvas o diagramas, el técnico preparado no solamente obtiene la respuesta cuantitativa a su problema, sino que recibe un intenso estímulo en los procesos mentales inductivos y de relación entre factores y fenómenos, que derivarán sin duda, en base a la intuición y proceso integrador característicos de la mente humana, en una fecunda potenciación de su capacidad creativa y de concepción de nuevas formas y sistemas, de los que tan rica es en posibilidades la construcción mixta actual.

\section{EL ORDENADOR COMO SOPORTE DE LOS NUE- VOS METODOS DE ANA- LISIS}

Además de la fecunda dialéctica entre teoría y práctica comentada en el apartado precedente, interesa resaltar, desde el punto de vista de la construcción mixta (o, más ampliamente, de la ciencia de las estructuras), otra dialéctica no menos interesante: la existente entre teoría y medios de cálculo. Si es cierto que la mayor parte de los modelos teóricos de última creación carecen de sentido sin el soporte del ordenador (y ello no sólo por razones de tiempos de cálculo, exactitud, etc., sino porque los propios formalismos están de hecho adaptados a la "lógica" de dicho instrumento), no es menos cierto que el plantearse modelos teóricos progresivamente más ambiciosos, potentes $y$ complejos ha estimulado una amplia actividad creativa de "software" (programación), y ha contribuido a la difusión y utilización del ordenador como instrumento para la investigación científica y para aplicaciones técnicas directamente productivas dentro del campo que se comenta, del mismo modo que ha sucedido en la generalidad de las actividades científicas y técnicas. En este sentido, a pesar de que estas últimas constituyen actualmente sólo una parte de los campos de utilización del cálculo electrónico, puede destacarse que el empleo de los primitivos computadores de la "primera generación" se ceñía inicialmente a la investigación científica y técnica; ésta, pues, aparece, al menos durante la génesis del ordenador, como un elemento inspirador inclu- so de la propia estructuración de dicho instrumento, por lo cual la dialéctica que se comenta es ciertamente muy profunda en ambos sentidos.

En el campo concreto de la ciencia de las estructuras, y haciendo referencia a las teorías desarrolladas en los últimos años, se observa una complejidad creciente en sus formalismos y un abandono de muchas líneas conceptuales que hasta un pasado reciente y todavía en muchos textos aparecían como fundamentales, pero que no han resistido los últimos cambios del "entorno" y la "selección natural" operativa también en este aspecto de la actividad humana. Ante este hecho irreversible no caben más ataques que los que se deriven de su mayor o menor eficacia, y no, en estricta lógica, los que apelen a la mayor o menor dificultad de adaptación a las nuevas teorías y formalismos. No obstante, hay que destacar el hecho de que la complejidad de las mismas sólo necesita ser asumida, a efectos de su aplicación práctica (naturalmente, el investigador teórico deberá moverse continuamente en ese campo), "una sola vez" y por un número limitado de miembros de un equipo, ya que, una vez elaborado un programa o sistema de programas de ordenador en base a la teoría en cuestión, la utilización de los mismos no tiene en principio porqué resultar desmesuradamente compleja. 
Para justificar esta afirmación, ya en el campo específico de la construcción mixta, se hace referencia al ejemplo indicado en la figura V.1, correspondiente al análisis de una sección de medio tablero de un puente mixto, de la que se indican los valores de las tensiones normales de servicio, diagrama momentos-curvaturas esquemático y diagrama de interacción flector-cortante, a título de ejemplo de resultados que pueden obtenerse con toda sencillez contando con un programa de análisis de secciones mixtas relativamente sofisticado (1). No cabe duda de que la obtención "manual» de los diagramas momentos-curvaturas y de interacción de dicha sección mixta resultaría una labor francamente ardua y laboriosa, lo suficiente probablemente (sobre todo cuando hay que repetirla para el resto de secciones de la pieza) como para abandonar dicha línea de cálculo y ceñirse a un $m$ é to $d$ o estrictamente elástico; no obstante, cuando el tiempo de obtención de dichos diagramas se reduce a segundos, y no requiriendo mayor suministro de información que la geometría y características de los materiales de la sección (prácticamente la misma cantidad de información que es necesaria para el cálculo de tensiones en régimen lineal), es indudable que desaparecen las objeciones de tipo práctico antes aludidas, aún cuando la teoría base del programa de cálculo sea relativamente complicada.

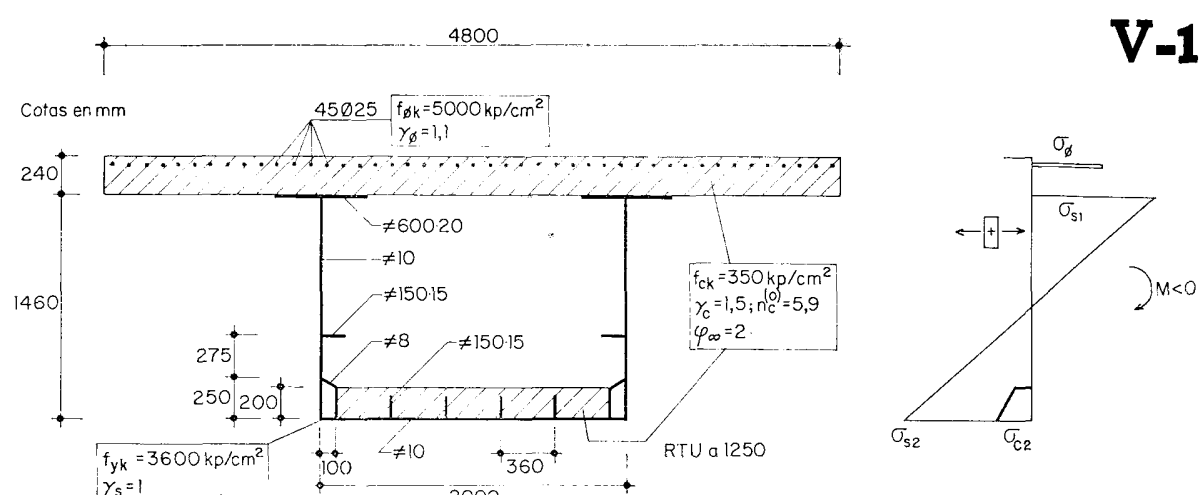

TENSIONES NORMALES DE SERVICIO (valores acumulados, en $\mathrm{kp} / \mathrm{cm}^{2}$ )
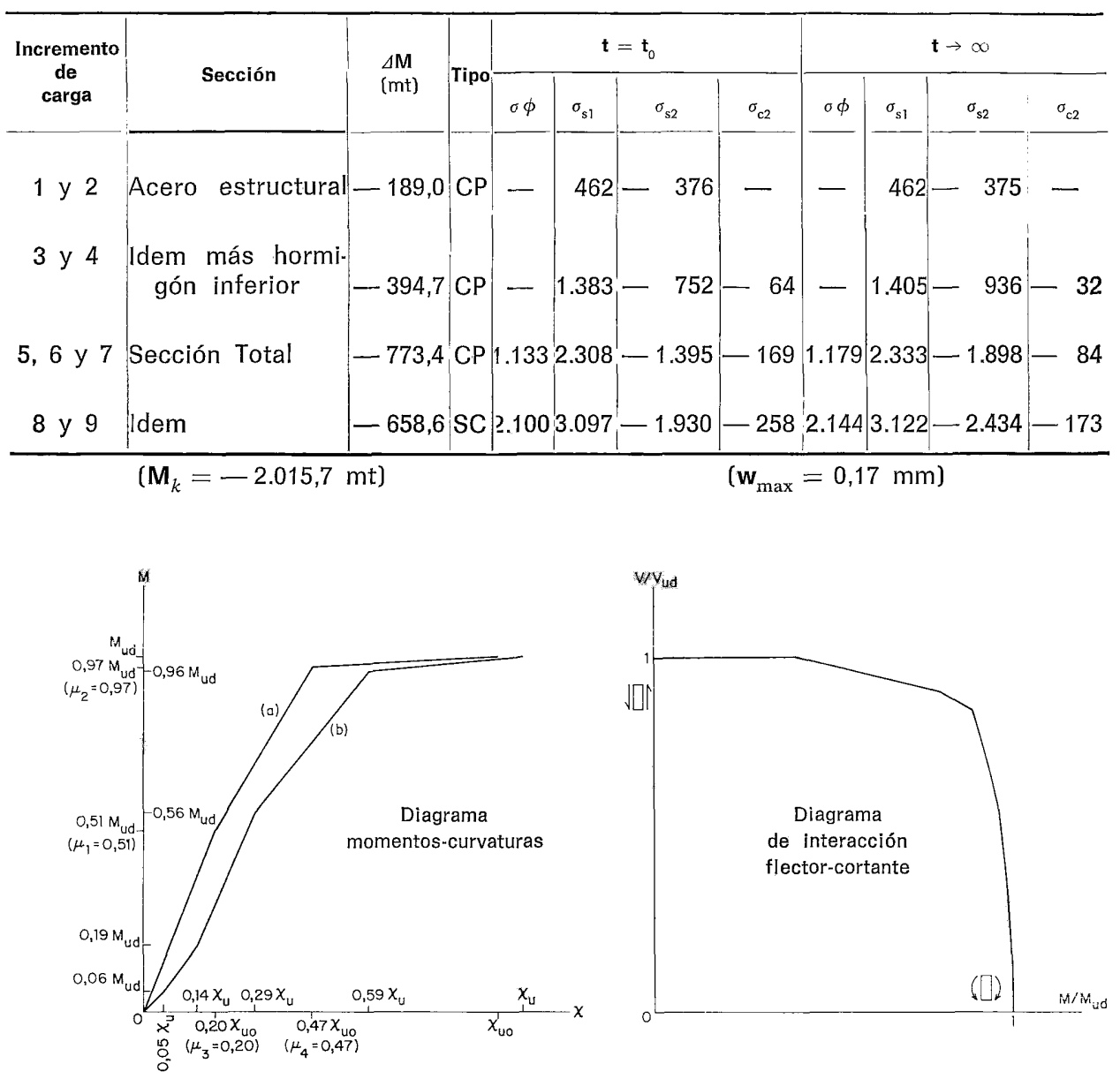

(Resistencias minoradas; diagramas tensión-deformación birrectilíneos; $\varepsilon_{\mathrm{cu}}^{\prime}=0,0035 ; \varepsilon_{\mathrm{su}}=0,025$ )

a) Sin predeformaciones de las secciones parciales; $\chi_{\mathrm{u} 0}=-0,00516 \mathrm{~m}^{-1}$.

b) Considerando el proceso real de aplicación de los esfuerzos parciales; $\chi_{\mathrm{u}}=1,07 \gamma_{\mathrm{u} 0}$.

En ambos casos, $M_{u d}=-3.126 \mathrm{mt} ; M_{\mathrm{D} 1 . \mathrm{d}} / M_{\mathrm{ud}}=1,03 ; \mathrm{M}_{\mathrm{ud}} / \mathrm{M}_{\mathrm{b}}=1,55>\gamma_{\mathrm{f}}$.

(1) En realidad la sección considerada está en el límite de aplicación del cálculo elastoplástico, por inestabilidad de chapas. Excede la limitación de esbeltez máxima de chapas de secciones compactas (en este sentido la sección es "semicompacta») en el recuadro superior de alma y en los recuadros de la chapa de fondo; pero la estabilidad de estos últimos está mejorada por su conexión al hormigón interno; y la sección eficaz del recuadro superior de alma coincide prácticamente con la sección completa del mismo, afectando además la diferencia entre ambas mínimamente (por su proximidad a la fibra neutra) al momento de agotamiento global de la sección. Por ello es válido, en este caso, controlar el estado límite de agotamiento mediante el cálculo elastoplástico o rígidoplástico usuales, en lo que respecta al agotamiento de las secciones. Pero las redistribuciones plásticas de momentos hiperestáticos deben limitarse adecuadamente; de hecho, es conveniente calcular este tipo de piezas en base a las envolventes elásticas de esfuerzos corregidas únicamente por coeficientes de redistribución de fisuración. 
Para el análisis sistemático de estructuras mixtas con arreglo a los criterios de cálculo en agotamiento y métodos no lineales en general, resulta actualmente indispensable de hecho (por razones de rigor, competitividad, eficacia, etc.) la utilización de métodos "computerizados" a todos los niveles, salvo que el campo de actuación se restrinja a una tipología definida y sencilla de piezas, en cuyo caso puede ser suficien. te la automatización parcial, limitada a los aspectos básicos del análisis, con equipos electrónicos de menor envergadura que los requeridos por una automatización "total" y de amplio espectro. No es posible comentar de modo breve la variedad completa de algoritmos y modalidades de programas susceptibles de integrarse en una biblioteca amplia de programas de cálculo y diseño de secciones y estructuras mixtas. Por ello, la exposición subsiguiente se limita a comentar e ilustrar mediante ejemplos dos elementos de cálculo particulares, seleccionados en función de su carácter relativamente sofisticado y novedoso, y de su interés específico para el análisis riguroso de estructuras mixtas importantes o complejas: en primer lugar, programas conversacionales de análisis de secciones mixtas en sus distintos aspectos (elástico, diferido, con fisuración, rígidoplástico, elastoplástico...), de gran interés para el proyecto de puentes o grandes estructuras mixtas, o para el análisis "singular» de piezas de utilización sistemática, cuyo comportamiento se quiere conocer a fondo en determinados casos típicos seleccionados; $y$, en segundo lugar, programas de análisis no líneal avanzado que permiten el cálculo en agotamiento de estructuras hiperestáticas considerando las leyes esfuerzos-deformaciones elastoplásticas de las secciones de las piezas, al objeto de fijar los niveles de redistribución de esfuerzos con total precisión y garantía, o de controlar el adecuado comportamiento plástico de una o varias piezas "testigo" de un conjunto o sistema de tipología definida (realizando auténticos ensayos de agotamiento simulados]. Limitando la exposición a estos dos temas, se dejan aparte otros tan interesantes como el comentario de programas "rígidos" (no conversacionales) para tabulación de parámetros deformativos y resistentes de sistemas de secciones de tipología definida; programas genéricos de análisis estructural lineal, adaptados a la problemática particular de las estructuras mixtas; programas de análisis diferido de estructuras mixtas, según diversos métodos; programas estructurales específicos para determinadas tipologías estructurales (emparrillados de vigas mixtas, emparrillados de cajones mixtos, etc.) ; programas complementarios para el análisis de piezas mixtas de edificación; programas complementarios para puentes mixtos (rigidización de chapas, cálculo y armado de la losa del tablero, etc.) ; sistemas de programas para el cálculo, dimensionamiento, delineación y medición enteramente automáticos de estructuras mixtas completas de edificación, etcétera.
Antes de pasar a especificar las características idóneas de un programa conversacional sofisticado para el análisis de secciones mixtas, es conveniente efectuar algunas observaciones respecto al equipo electrónico adecuado para este tipo de programa. Indudablemente, al ser preciso reunir en un mismo programa (o sistema segmentado de programas mutuamente enlazados) un conjunto de diversos algoritmos de análisis, juntamente con entradas/salidas muy casuísticas, controladas y diversificadas, no cabe pensar en microordenadores o equipos similares. En el otro extremo se encuentran los grandes equipos, los cuales permiten sin duda la realización de cálculos de la máxima complejidad; pero exigiendo a su vez una explotación sistemática que repercute, salvo en sistemas de utilización múltiple o compartida (con terminales adecuados para la representación gráfica que conviene a estos programas], es una gran rigidez de comunicación máquina-usuario, perdiéndose o limitándose una de las características que se consideran más interesantes para este tipo de programas: la de permitir al usuario un proceso de cálculo interactivo, rectificando datos y repitiendo cálculos a medida que progresa en su proceso de diseño, realizando éste en «diálogo" continuado con la máquina. Por todo ello, el equipo que se considera idóneo para la clase de programa que se menciona, es un equipo de tipo medio, manejable directamente por el propio proyectista sin excesivas complicaciones, y que permita al mismo tiempo la adecuada versatilidad de cálculo que requieren las secciones mixtas complejas. 
Como características deseables de un buen programa de esta naturaleza, se mencionan las siguientes:

a) Ser conversacional: es decir, efectuar las diferentes operaciones de entrada/salida, operaciones "lógicas" y de análisis, en función de órdenes transmitidas directamente por el usuario desde una consola, aceptando asimismo la definición "manual" de todo tipo de datos para el cálculo; lo cual no impide que, opcionalmente, parte o la totalidad de los datos se introduzcan codificados mediante tarjetas o cinta perforadas (por ejemplo) desde periféricos auxiliares, sie mpre a elección del usuario en cada instante. El programa debe controlar al máximo todas las entradas de datos y ejecutivas (órdenes de operaciones), advirtiendo los diversos errores y situaciones anómalas posibles, y devolviendo en todo caso el control al usuario para las rectificaciones oportunas. El conjunto de operaciones posibles debe disponerse en ciclo cerrado: una vez ejecutada cualquiera de ellas (excepto, naturalmente, la que realice el final del programa, retornando al control por el sistema operativo), debe volverse a un status en que se pueda ordenar cualquiera de ellas de nuevo; en efecto, tal como se ha indicado anteriormente, el proceso de diseño de las secciones mixtas complejas es generalmente un proceso de aproximaciones sucesivas, que exige rectificaciones y repeticiones hasta llegar a una solución optimizada; por otra parte, la posibilidad de efectuar de manera rápida y sencilla la rectificación parcial de todo tipo de datos geométrico-resistentes, es de gran utilidad para el tratamiento en cadena de una secuencia de secciones similares entre sí, como suelen ser las diferentes secciones de un puente o estructura mixta. Otra característica interesante es la de insertar adecuados comentarios a las entradas de datos y órdenes, o permitir que dichos comentarios sean solicitados por el usuario, de forma que la comunicación con el programa sea en todo momento lo más "natural" posible, permitiendo al usuario concentrarse en el diseño y optimización de soluciones. b) Efectuar salidas de resultados diversificadas y comentadas. Es altamente deseable disponer de periféricos para la salida gráfica de secciones, distribuciones tensionales, diagramas de interacción de esfuerzos, diagramas esfuerzos - deformaciones, etc. Asimismo, es de gran utilidad la posibilidad de que el programa efectúe memorias autoexplicativas de datos y de resultados de los diversos cálculos, lo cual permite obviar o reducir la nada despreciable tarea de elaboración de las memorias de cálculo anejas al proyecto. Todas estas salidas deben poder ser ordenadas por el usuario en el momento en que éste considere terminada 0 definitiva cada fase de cálculo; todas las salidas de tanteos previos propios del proceso de diseño deben efectuarse por consola, mediante una pantalla o elemento adecuado.

c) Es muy conveniente disponer de un banco de almacenamiento de secciones en una memoria periférica (disco o cinta magnética), donde sea posible conservar por tiempo arbitrario la información relativa a un cierto número de secciones, accediendo o actuando sobre ella (modificaciones, borrados parciales, etc.) cuando sea preciso. Esta memoria auxiliar de secciones puede asimismo servir para la comunicación con otros programas; por ejemplo, los que efectúan el análisis estructural. 
d) Incorporar los siguientes algoritmos, todos ellos referidos a una sección de tipología prácticamente arbitraria o con limitaciones mínimas, tales como las de ceñirse a secciones con un eje de simetría y constituidas por rectángulos de distintos materiales (por lo demás, las combinaciones de secciones parciales y de los diferentes materiales deben ser enteramente arbitrarias):

- Cálculo de constantes estáticas en distintas variantes: sección total y/o secciones parciales; constantes estáticas "iniciales" o "diferidas" (método de la sección ideal); con o sin fisuración (definiendo el estado de fisuración en distintas configuraciones: flexión pura positiva o negativa, tracción simple, axil excéntrico de excentricidad dada, etc.).

- Análisis tensional lineal o cuasi - lineal (considerando deformaciones diferidas y/o fisuración), para sucesivos incrementos de esfuerzos de diversa $\mathrm{n}$ a t u raleza: momentos, axiles centrados o excéntricos, cortantes; pretensado; retracción; esfuerzos térmicos, etcétera. Es preciso actuar como mínimo con dos distribuciones tensionales (inicial y a tiempo infinito), modificándolas para cada incremento de carga según la naturaleza del mismo (permanente, sobrecarga rápida...) y las características reológicas de los hormigones de la sección. Asimismo, cada incremento de esfuerzos puede afectar a la sección total o a una determinada sección parcial, lo cual $\mathrm{d}$ e b e, naturalmente, ser tenido en cuenta para su incorporación al estado tensional de la sección.

- Obtención de esfuerzos de agotamiento (flectores positivo y negativo, c o r tante, axiles últimos) por el método rígido-plástico y elastoplástico.

- Obtención de diagramas de interacción entre esfuerzos últimos: flector-cortante, con o sin axil constante aplicado simultáneamente; flectoraxil, con o sin esfuerzo cortante.

- Determinación de leyes esfuerzos - deformaciones elastoplásticas: diagramas momentos-curvaturas, teniendo en cuenta la influencia de presolicitaciones, pretensados, esfuerzo axil constante simultáneo, etcétera; diagramas axil-elongación (de menor interés), también dependientes en general de las posibles predeformaciones y momento aplicado simultáneamente.

- Otros algoritmos de interés son los relativos al cálculo de la conexión, abolladura, torsiones, etc.
Como ejemplo de algunos de los resultados que son obtenibles con toda sencillez mediante un programa de esta naturaleza pueden verse los indicados en las figuras V.1 y V.2 (1). Los valores indicados en esta última (fig. V.2) sirven de base para el ejemplo de utilización de los programas de análisis estructural elastoplástico, los cuales son comentados a continuación.

Para el análisis no lineal riguroso de estructuras mixtas que involucran secciones complejas, es generalmente necesario operar con diagramas momentos-curvaturas trilineales o multilineales, variables además generalmente a lo largo de las piezas y según el signo de la flexión (v. figuras V.1 y V.2). Si las piezas están sometidas a flexión compuesta, la influencia de los axiles exteriores (2) sobre las leyes momentos-curvaturas, e incluso la propia no linealidad de las leyes axilelongación y la no linealidad "geométrica" (fenómenos de inestabilidad) pueden tener que ser incorporadas al análisis, para la determinación correcta de la carga última. En cuanto al esfuerzo cortante, si los correspondientes términos de deformaciones de las piezas pueden despreciarse frente a los de flexión (caso de relaciones luz/canto no relativamente pequeñas, y conexión no excesivamente flexible), puede entonces limitarse su efecto a una reducción del momento de agotamiento de la sección, considerada a posteriori o mediante un retoque del correspondiente diagrama momentos-curvaturas; de lo contrario, la deformabilidad de cortante de las piezas, habida cuenta incluso de su fase no lineal, debe ser incorporada al análisis.

(1) Las secciones consideradas en ambos ejemplos responden a tipologías comentadas en el ap. IV.

(2) Excluido el pretensado, el cual, en régimen elastoplástico, se introduce directamente como una predeformación parcial, al obtener los diagramas momentos-curvaturas. 


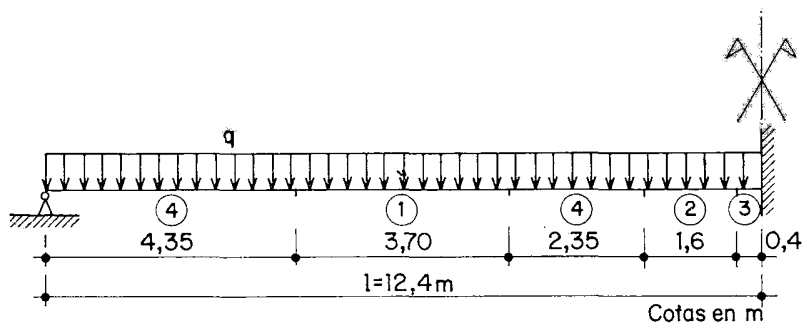

(a) Hormigón in situ: $\gamma_{\mathrm{c}}=1,5$.

(b) Hormigón de taller: $\mathrm{f}_{\mathrm{ck}}=450$, $\gamma_{\mathrm{c}}=1,4$.

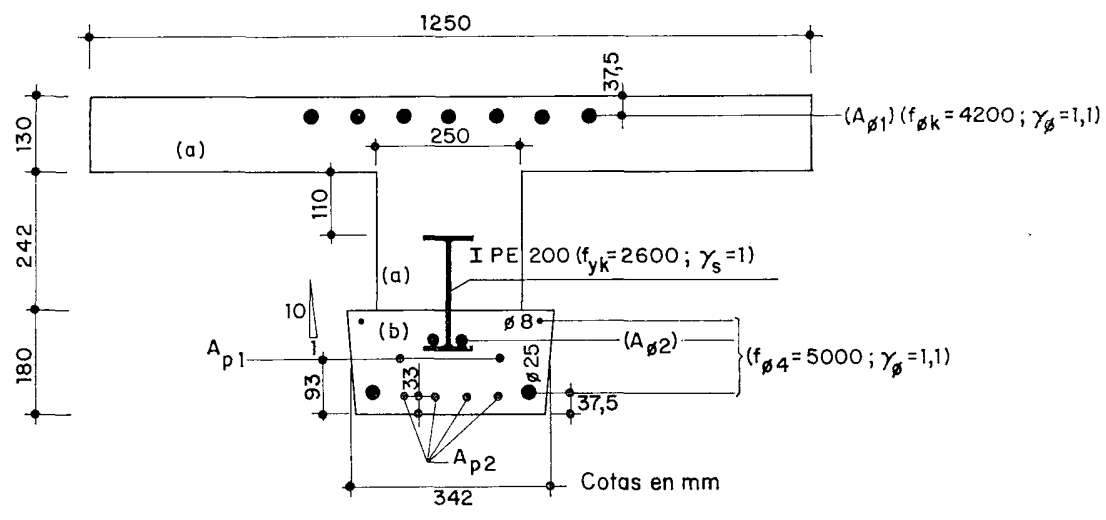

SECCION DE CALCULO ESQUEMATICA.

Resistencias, en $\mathrm{kp} / \mathrm{cm}^{2}$

$A_{\mathrm{p} 1}$ y $\mathbf{A}_{\mathrm{p} 2}: \mathbf{f}_{\mathrm{pk}}=16.000$; $\gamma_{\mathrm{p}}=1,15$;

tesado $=120 \mathrm{t}$ sin hormigón in situ (perfil metálico más suela armada).

\begin{tabular}{c|c|c|c|c}
\hline Sección tipo & $\mathbf{A} \phi_{1}$ & $\mathbf{A} \phi_{2}$ & $\mathbf{A}_{\mathrm{D} 1}$ & $\mathbf{A}_{\mathrm{D} 2}$ \\
\hline 1 & - & $2 \varnothing 20$ & $3,1 \mathrm{~cm}^{2}$ & $6,2 \mathrm{~cm}^{2}$ \\
2 & $6 \varnothing 25$ & - & $n$ & $n$ \\
$3\left(^{*}\right)$ & $"$ & - & - & - \\
4 & - & - & $3,1 \mathrm{~cm}^{2}$ & $6,2 \mathrm{~cm}^{2}$ \\
\hline
\end{tabular}

- Sección de empotramiento en soporte de hormigón; $A_{p}=0$, y $f_{c k}=175$ incluso en la zona (b).

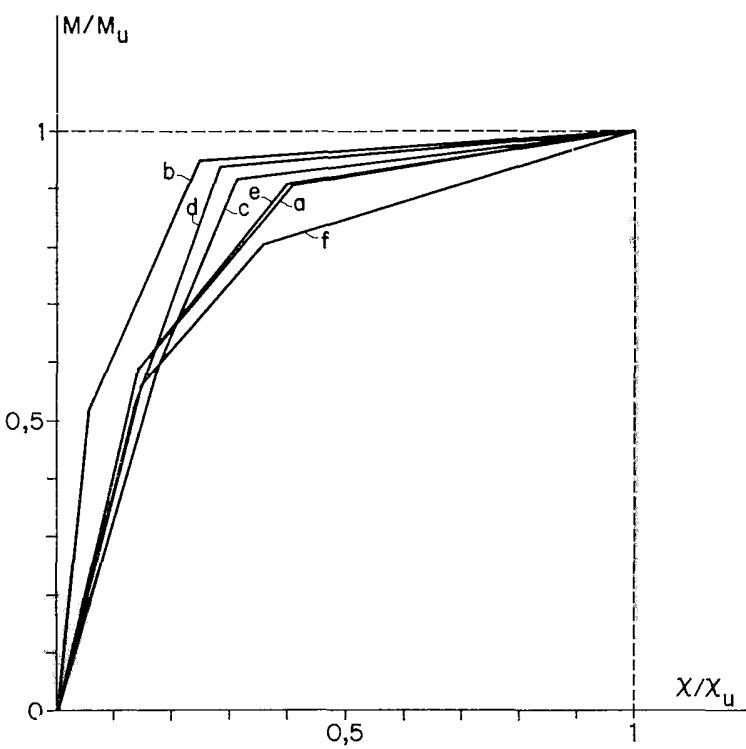

Diagramas momentos-curvaturas trilineales reducidos.

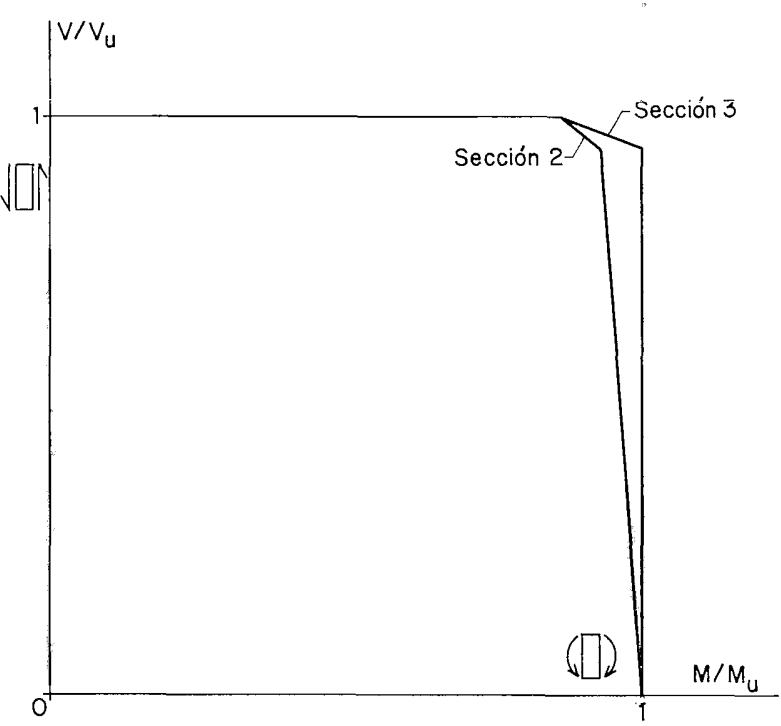

Diagramas de interacción flector-cortante.

\begin{tabular}{|c|c|c|c|c|c|c|c|c|}
\hline Gráfico & Sección & Signo flexión & $\begin{array}{c}M_{u} \\
(m t)\end{array}$ & $\left(\begin{array}{c}x_{u} \\
\left(m^{-1}\right)\end{array}\right.$ & $\mu_{1}$ & $\mu_{2}$ & $\mu_{3}$ & $\mu_{4}$ \\
\hline a & 1 & + & 100,7 & 0,01242 & 0,614 & 0,910 & 0,160 & 0,411 \\
\hline b & 2 & + & 114,3 & 0,03366 & 0,520 & 0,950 & 0,053 & 0,246 \\
\hline c & 2 & - & $-62,4$ & $-0,01972$ & 0,586 & 0,921 & 0,172 & 0,312 \\
\hline d & 3 & - & $-56,1$ & $-0,02367$ & 0,520 & 0,942 & 0,136 & 0,282 \\
\hline e & 4 & + & 98,3 & 0,01348 & 0,593 & 0,909 & 0,141 & 0,401 \\
\hline$f$ & 4 & - & $-13,1$ & $-0,02949$ & 0,562 & 0,803 & 0,144 & 0,355 \\
\hline
\end{tabular}

NOTA. Cálculo con resistencias características y diagramas tensión-deformación birrectilíneos $\left(\varepsilon_{\mathrm{cu}}^{\prime}=0,0035, \varepsilon_{\mathrm{su}}=0,025\right)$. 
En el caso de emparrillados analizados elastoplásticamente es asimismo preciso representar la deformabilidad torsional mediante el correspondiente diagrama torsoresgiros unitarios de torsión; y deben tenerse en cuenta, en su caso, las interacciones con las flexiones simultáneas.

El análisis no lineal riguroso de las estructuras mixtas complejas aparece, pues, como un problema difícil, para el cual no son generalmente válidos modelos simplificados basados en diagramas momentos-curvaturas birrectilíneos y en la ausencia de otros efectos no lineales, salvo cuando se limita el alcance del análisis al caso de piezas de tipología y cargas sencillas, exclusivamente en flexión simple, de las que se pretende tan sólo obtener una idea de las redistribuciones posibles de momentos hiperestáticos y no una determinación completa del comportamiento no lineal (1). Para esto último, es decir, para la incorporación de diagramas esfuerzos-deformaciones multilineales arbitrarios, y otros efectos no lineales (no linealidad geométrica), a pesar de la dificultad indudable que ello conlleva, existen algoritmos sistemáticos, adecuados para su programación en ordenador, generalmente con estructuración un tanto similar a la de los programas lineales (siendo entonces posible el aprovechamiento de parte de los subprogramas de estos últimos). Pero su utilización práctica está en la actualidad bastante limitada, en parte por la menor difusión de estos algoritmos, y también por inconvenientes de orden práctico, tales como:
- Mayor número de datos necesarios para definir el comportamiento no lineal de las secciones de las piezas. Ello obliga a cálculos previos extensos (determinación y esquematización de leyes esfuerzosdeformaciones), muy sensibles además a las variaciones de las armaduras, refuerzos, etc., lo cual determina que el número de secciones distintas a considerar pueda llegar a ser particularmente elevado; y que cualquier modificación posterior de las secciones, a la vista de los resultados del análisis estructural no lineal, pueda invalidar este análisis. Ello obliga a un predimensionamiento muy afinado y prolijo; y, muy probablemente, a una o más repeticiones del análisis estructural no lineal, cuando sea preciso optimizar la pieza o estructura.

- Aparte de esto, cada análisis estructural no lineal es ya de por sí más lento que un análisis según procedimientos elásticos, ya que son precisas generalmente como mínimo de tres a cinco iteraciones globales.

- Por otra parte, la no validez del principio de superposición inherente a todo método no lineal, obliga a calcular la estructura bajo las acciones totales de la misma, no siendo posible analizar por separado diversas configuraciones de cargas permanentes y sobrecargas y superponer después los resultados; ni tampoco extrapolar linealmente los resultados obtenidos para una configuración dada de cargas, cuando todas ellas se multiplican por un factor o coeficiente de mayoración común. Ello obligaría en rigor a aumentar enormemente, en muchos casos, el número de hipótesis de carga a considerar. Por otra parte, debido a la modificación iterativa de la matriz de rigidez de la estructura durante el proceso de resolución de cada una de las hipótesis de carga, no es posible analizar éstas conjuntamente (ello sí es posible, por el contrario, dentro del análisis elástico y permite acelerar notablemente el análisis de estructuras con gran número de alternancias o de posiciones distintas de sobrecargas).

Por todo ello, en la actualidad, la aplicación práctica de los métodos no lineales no es viable con carácter sistemático, sino que se circunscribe a estudios de investigación o a cálculos singulares, tales como la determinación de coeficientes de redistribución de estructuras importantes en algunas configuraciones de carga seleccionadas (para la subsiguiente modificación aproximada de las envolventes elásticas de esfuerzos); o en sustitución (favorable en tiempos y costes) de ensayos de piezas especiales o de tipologías nuevas, cuyo comportamiento no lineal se desea conocer con rigor. A este objetivo corresponde el ejemplo indicado en la figura V.3 (cuyas secciones aparecen definidas y estudiadas en la figura V.2).

(1) No se pretende menoscabar la utilidad de estos modelos simplificados, que es muy estimable a los efectos indicados, sino resaltar la dificultad del análisis no lineal riguroso de las estructuras mixtas complejas. 
Para la aplicación sistemática de los métodos no lineales, a pesar de que el aspecto teórico de los mismos puede en la actualidad considerarse esencialmente resuelto, será preciso probablemente esperar a una generación futura de ordenadores en los cuales la capacidad de memoria interna y rapidez de cálculo se hayan mejorado (en características y coste) sustancialmente respecto a los actuales ordenadores. Esta es, pues, una situación en que la teoría camina por delante de los medios de cálculo, en su dialéctica mutua.

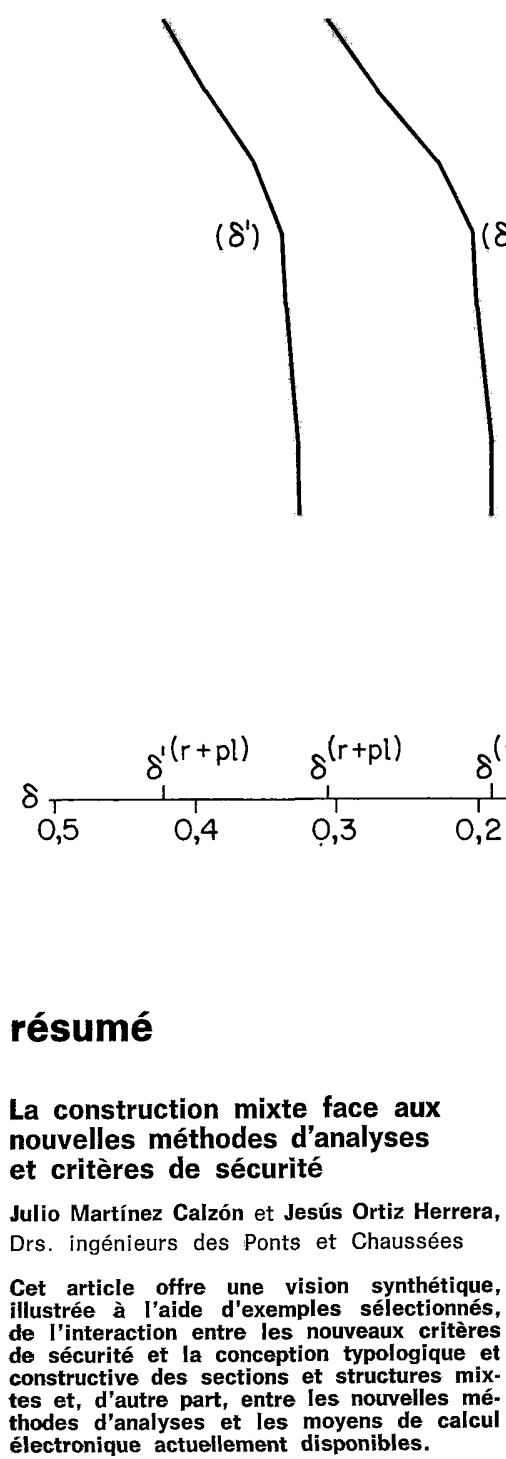

\section{summary}

Compound steel and concrete construction and the new methods of analysis and safety criteria

Julio Martínez Calzón and Jesús Ortiz Herrera, Dr. Civil Engineers

This paper includes: a comprehensive review, illustrated with suitable examples, of the interaction of the new safety criteria and the methods of design and construction of compound structures; and an account of of compound structures; and an account recent methods of electronic analysis
currently available.

$q=$ carga aplicada el carácter variable de la inercia de la pieza,

$\left(\delta^{\prime}\right)$ - redistribuciones respecto a $\mathbf{M}_{0, \text { el }}^{\prime}=\mathbf{q l}^{2} / 8$ (momento de empotra= flecha en el centro de la luz:

(2) - análisis con fisuración teórica y comportamiento elastoplástico (según los diagramas momentos-curvaturas trilineales indicados en la figura V.2).

$q, t / m$

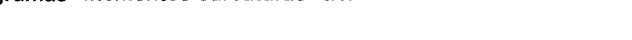

